\title{
A novel robust nomogram based on preoperative hemoglobin and albumin levels and lymphocyte and platelet counts (HALP) for predicting lymph node metastasis of gastric cancer
}

\author{
Xu Wang ${ }^{\#} \wedge$, Qijin He ${ }^{\#}$, Huixi Liang, Jiani Liu, Xin Xu, Kui Jiang^, Jie Zhang^ \\ Department of Gastroenterology and Hepatology, Tianjin Medical University General Hospital, Tianjin Institute of Digestive Diseases, Tianjin Key \\ Laboratory of Digestive Diseases, Tianjin, China \\ Contributions: (I) Conception and design: X Wang, Q He; (II) Administrative support: J Zhang, K Jiang; (III) Provision of study materials or patients: \\ J Zhang, K Jiang, X Wang; (IV) Collection and assembly of data: Q He, H Liang, J Liu, X Xu; (V) Data analysis and interpretation: X Wang, Q He; (VI) \\ Manuscript writing: All authors; (VII) Final approval of manuscript: All authors. \\ \#These authors contributed equally to this work. \\ Correspondence to: Jie Zhang. Department of Gastroenterology and Hepatology, Tianjin Medical University General Hospital, Tianjin Institute of \\ Digestive Diseases, Tianjin Key Laboratory of Digestive Disease, No. 154 Anshan Road, Tianjin 300052, China. Email: Zhangjie_xhk@tmu.edu.cn; \\ Kui Jiang. Department of Gastroenterology and Hepatology, Tianjin Medical University General Hospital, Tianjin Institute of Digestive Diseases, \\ Tianjin Key Laboratory of Digestive Disease, No. 154 Anshan Road, Tianjin 300052, China. Email: jiangkui@tmu.edu.cn.
}

\begin{abstract}
Background: Accurate assessment of lymph node status in gastric cancer (GC) patients can help to select appropriate treatment strategies for GC, but the diagnostic accuracy of conventional methods needs to be improved. The aim of this study was to investigate the predictive value of preoperative hemoglobin and albumin levels and lymphocyte and platelet counts (HALP) on lymph node status in GC patients and to construct a risk prediction model.

Methods: This study retrospectively analyzed the clinicopathological characteristics of 349 patients with GC who underwent radical gastrectomy, among which 250 patients were recruited in the training cohort and 99 patients in the independent validation cohort. Significant risk factors in univariate analysis were further identified as independent variables in multivariate logistic regression analysis, which were then incorporated and presented in a nomogram. Receiver operating characteristic (ROC) curves, Calibration curve and decision curve analysis (DCA) curves were used to evaluate the discrimination, prediction accuracy and clinical effectiveness of the model

Results: Multifactorial logistic regression analysis showed that alcohol use ( $\mathrm{OR}=2.203, \mathrm{P}=0.036)$, Depth of invasion ( $\mathrm{OR}=7.756, \mathrm{P}<0.001)$, differentiation ( $\mathrm{OR}=2.252, \mathrm{P}=0.018)$, carcinoembryonic antigen (CEA) $(\mathrm{OR}=2.443, \mathrm{P}=0.017)$, carbohydrate antigen 19-9 (CA199) $(\mathrm{OR}=2.715, \mathrm{P}=0.008)$ and HALP $(\mathrm{OR}=2.276$, $\mathrm{P}=0.032)$ were independent risk factors for lymph node metastasis (LNM) in GC. We used these factors to construct a nomogram for predicting LNM in GC patients, and the ROC curves showed good discrimination of the model with AUC values of 0.854 (training cohort) and 0.868 (validation cohort), respectively, and the calibration curves showed good predictive ability of the nomogram, in addition to the DCA curves results showed the clinical usefulness of the model.
\end{abstract}

Conclusions: In conclusion, we established a nomogram for predicting LNM in patients with GC.

Keywords: Gastric cancer (GC); lymph node metastasis (LNM); nomogram; risk factors

\footnotetext{
^ ORCID: Xu Wang, 0000-0001-8714-4856; Qijin He, 0000-0003-3203-5331; Kui Jiang, 0000-0001-8852-5562; Jie Zhang, 0000-00032140-3231.
} 
Submitted Aug 16, 2021. Accepted for publication Oct 25, 2021.

doi: 10.21037/jgo-21-507

View this article at: https://dx.doi.org/10.21037/jgo-21-507

\section{Introduction}

Gastric cancer (GC) is one of the most serious malignant tumors with the fifth highest incidence rate and the third highest fatality rate in the world (1). Despite substantial progress in the diagnosis and treatment of GC in recent years, the overall five-year survival rate is still less than $20 \%$ due to local recurrence or metastasis after resection of primary GC (2). The most important factor affecting the prognosis of GC patients is lymph node metastasis (LNM), and it is prone to recurrence after surgery (3). Even the LNM rate of early GC is $3-25 \%$, and for patients with advanced GC, the LNM rate is as high as $80-90 \%$ (4). Accurate determination of LNM plays a crucial role in the choice of treatment modality. Therefore, accurate prediction and assessment of the risk of LNM is important for the choice of treatment $(5,6)$.

However, there is no method to accurately determine LNM in GC. Multilayer spiral CT is a commonly used imaging test to assess LNM, with a sensitivity of $55.3 \%$ and a specificity of $86.0 \%$ (7). Several new molecular biomarkers have been found to be useful for predicting LNM in GC, but their high cost and complex technology usually make them less likely to be used in the clinical setting $(8,9)$. Therefore, the search for a reliable and affordable preoperative biomarkers to assess the lymph node status of GC is still ongoing.

In recent years, some indicators derived from peripheral blood, such as neutrophil-to-lymphocyte ratio (NLR) and platelet-to-lymphocyte ratio (PLR), have been studied and proven to be predictive of LNM in GC (10-12). However, these indicators can only reflect the inflammatory status of the body to some extent. HALP score is a comprehensive score that combines hemoglobin, albumin, lymphocyte and platelet count, which can more comprehensively assess the nutritional and immune status of patients. It has been reported in the literature that HALP has a predictive effect on prognosis in GC (13). However, no study has been conducted to date to investigate the predictive value of HALP on the LNM of patients with GC. The aim of this study was to investigate the predictive value of HALP on LNM in GC and to develop a new nomogram for more accurate assessment of LNM in GC patients.
We present the following article in accordance with the TRIPOD reporting checklist (available at https://dx.doi. org/10.21037/jgo-21-507).

\section{Methods}

\section{Patients}

Clinical data of 349 GC patients who underwent radical GC surgery at Tianjin Medical University General Hospital from May 2018 to May 2020, among which 250 patients were recruited in the training cohort and 99 patients in the independent validation cohort. Inclusion criteria: (I) all patients with GC underwent total or subtotal gastrectomy with D1 or D2 lymph node dissection; (II) complete postoperative pathology reports and a clear diagnosis of GC; (III) patients with primary treatment and no preoperative anticancer therapy, such as chemotherapy or radiotherapy; (IV) no concurrent and heterochronous malignancies; (V) estimated life expectancy $\geq 3$ months after surgery. Patients with one of the following characteristics were excluded: (I) number of surgically cleared lymph nodes $<15$; (II) with significant infection, bleeding and other hematologic diseases; (III) history of gastrectomy; (IV) patients with incomplete data; (V) autoimmune diseases or hematologic diseases. The study was conducted in accordance with the Declaration of Helsinki (as revised in 2013). The study was approved by the Ethical Committee of Tianjin Medical University General Hospital (No.: IRB2021-WZ-114) and individual consent for this retrospective analysis was waived.

\section{Parameters}

We recorded the clinical characteristics of patients in the study, including age, gender, history of smoking and alcohol use, the presence of hypertension, diabetes, and coronary heart disease. Blood test indicators included hemoglobin, lymphocyte, platelet, total protein (TP), albumin (ALB), glutamic-oxalacetic transaminase (AST), glutamic-pyruvic transaminase (AST), lactate dehydrogenase (LDH), alkaline phosphatase (ALKP), total bilirubin (Tbil), direct bilirubin (Dbil), the concentrations of serum carcinoembryonic antigen (CEA) and carbohydrate antigen 19-9 (CA199), 
prothrombin time (PT), fibrinogen (FIB), activated partial thromboplastin time (APTT), thrombin time (TT) were calculated from the above indicators. LST was defined as follow: $\mathrm{LST}=\mathrm{AST}(\mathrm{U} / \mathrm{L}) / \mathrm{AST}(\mathrm{U} / \mathrm{L})$. HPR was defined as follow: HPR $=$ Hemoglobin $(\mathrm{g} / \mathrm{L}) /$ Platelets $\left(10^{9} / \mathrm{L}\right)$. HALP, the value of which was defined as follow: HALP $=$ Hemoglobin $(\mathrm{g} / \mathrm{L}) \times$ Albumin $(\mathrm{g} / \mathrm{L}) \times$ Lymphocytes $\left(10^{9} / \mathrm{L}\right) /$ Platelets $\left(10^{9} / \mathrm{L}\right)$. All patients' peripheral venous blood specimens were collected and tested within 1 week before surgery. Postoperative pathology results including tumor location, differentiated type, invasive depth were independently reviewed by two senior doctors in the pathology department. All patients had biopsy pathology confirmed as GC, and the cancers were classified as differentiated (papillary carcinoma, well differentiated tubular adenocarcinoma, and moderate tubular adenocarcinoma) or undifferentiated (poorly differentiated tubular adenocarcinoma, signet ring cell carcinoma, and mucinous adenocarcinoma) according to the Japanese GC and the World Health Organization GC classification (3rd edition) (14).

\section{Statistical analysis}

Statistical analysis was performed using IBM SPSS 23.0 and $\mathrm{R}$ (version $\mathrm{x} 64$ 4.0.4). Categorical variables were expressed as number and percentages and analyzed by $\chi^{2}$ test for univariate analysis. Continuous variables with normal distribution were expressed by mean \pm standard deviation (SD) and analyzed using the independent-samples $t$-test. Continuous variables with non-normal distribution were expressed by median and interquartile range P50 (P25P75) and analyzed by Mann-Whitney U tests. Significant factors obtained in univariate analysis were subsequently entered into multivariate logistic regression analyses to identify the independent variables associated with LNM in patients with GC. For continuous variables with significant differences between groups, we transformed these variables to categorical variables according to cut-off points, which were defined as the maximum value of the Youden index. The independent risk factors were used for the construction of the nomogram prediction model on the "rms" package of the $\mathrm{R}$ software. The prediction model formed in the training cohort was applied to the validation cohort to validate and evaluate the predictive power of the nomogram. The calibration of the nomogram was performed internally in the training cohort and externally in the validation cohort, using a calibration plot with bootstrap sampling $(n=1,000)$.
Discrimination (the ability of a nomogram to separate GC patients with LNM) was quantified by means of the area under the receiver operating characteristic (ROC) curve (AUC or C-index). Decision curve analysis (DCA) was applied to evaluate the clinical usefulness of the prediction model. All analyses were performed as two-tailed tests with a significance level of $5 \%$, meaning that $\mathrm{P}<0.05$ was considered to indicate a statistically significant difference.

\section{Results}

\section{Clinical characteristics of GC patients}

According to the inclusion and exclusion criteria, 349 patients were included in final analysis, all of whom were randomly divided into the training cohort $(\mathrm{n}=250)$ and validation cohort $(n=99)$ in the ratio $7: 3$. The percentage of lymph node metastases in the two cohorts were $60.8 \%$ $(n=152)$ and $63.6 \%(n=63)$. The training cohort appeared to have higher levels of ALKP, and the remaining basic clinical characteristics did not differ significantly between the training and validation cohorts, confirming that the training and validation cohorts had similar baseline data. In the training cohort, the mean age was $64.8 \pm 10.1$ years and $183(73.2 \%)$ of the patients were male. The GC was located in the lower part of the stomach $(51.6 \%)$ in most cases, followed by the middle portion (35.6\%), upper portion $(12.8 \%)$. In histology differentiation, the ratios of differentiated and undifferentiated grade were $51.2 \%$ and $48.8 \%$, respectively. The postoperative results confirmed lymphatic metastasis in 152 cases $(60.8 \%)$, including 36 cases $(14.4 \%)$ in stage $\mathrm{N} 1,38$ cases $(15.2 \%)$ in stage $\mathrm{N} 2$, and 78 cases $(31.2 \%)$ in stage N3. The clinicopathological characteristics of GC patients in the training cohort and validation cohort are shown in Table 1 .

\section{Univariate analysis of factors associated with LNM in the training cohort}

In the training cohort, 250 patients were divided into $\operatorname{LNM}(+)$ group $(\mathrm{n}=152)$ and $\operatorname{LNM}(-)$ group $(\mathrm{n}=98)$ according to the presence or absence of LNM. According to the univariate analysis, alcohol use, weight loss, depth of invasion, differentiation, PT, CEA, CA199, HPR and HALP were significantly correlated with LNM (all with $\mathrm{P}<0.05$ ). The above risk factors with $\mathrm{P}<0.05$ were included in the multivariate analysis. The cut-off points of PT, CEA, CA199, HPR, and HALP were obtained according to the optimal 
Table 1 Characteristics of patients in the training and validation cohorts

\begin{tabular}{|c|c|c|c|}
\hline \multirow{2}{*}{ Variable } & Training cohort & Validation cohort & \multirow{2}{*}{$\mathrm{P}$} \\
\hline & $\mathrm{n}=250$ & $\mathrm{n}=99$ & \\
\hline Age (years) & $64.8 \pm 10.1$ & $65.2 \pm 9.2$ & 0.691 \\
\hline Gender & & & 0.398 \\
\hline Male & $183(73.2 \%)$ & $68(68.7 \%)$ & \\
\hline Female & $67(26.8 \%)$ & $31(31.3 \%)$ & \\
\hline Smoking & & & 0.528 \\
\hline No & $127(50.8 \%)$ & $54(54.5 \%)$ & \\
\hline Yes & $123(49.2 \%)$ & $45(45.5 \%)$ & \\
\hline \multicolumn{4}{|l|}{ Alcohol use } \\
\hline No & $172(68.8 \%)$ & $67(67.7 \%)$ & \\
\hline Yes & $78(31.2 \%)$ & $32(32.3 \%)$ & \\
\hline Diabetes & & & 0.937 \\
\hline No & $196(78.4 \%)$ & 78 (78.8\%) & \\
\hline Yes & $54(21.6 \%)$ & $21(21.2 \%)$ & \\
\hline \multicolumn{2}{|c|}{ Coronary heart disease } & & 0.861 \\
\hline No & 209 (83.6\%) & $82(82.8 \%)$ & \\
\hline Yes & $41(16.4 \%)$ & $17(17.2 \%)$ & \\
\hline Hypertension & & & 0.445 \\
\hline No & $148(59.2 \%)$ & $63(63.6 \%)$ & \\
\hline Yes & $102(40.8 \%)$ & $36(36.4 \%)$ & \\
\hline Location & & & 0.303 \\
\hline Upper $1 / 3$ & $32(12.8 \%)$ & $15(15.2 \%)$ & \\
\hline Middle 1/3 & $89(35.6 \%)$ & $42(42.4 \%)$ & \\
\hline Low $1 / 3$ & $129(51.6 \%)$ & $42(42.4 \%)$ & \\
\hline Weight loss & & & 0.653 \\
\hline No & $148(59.2 \%)$ & $56(55.6 \%)$ & \\
\hline Yes & $102(40.8 \%)$ & $43(43.4 \%)$ & \\
\hline Depth of invasion & & & 0.530 \\
\hline $\mathrm{T} 1 / \mathrm{T} 2$ & $100(40.0 \%)$ & $36(36.4 \%)$ & \\
\hline $\mathrm{T} 3 / \mathrm{T} 4$ & $150(60.0 \%)$ & $63(63.6 \%)$ & \\
\hline Histological type & & & 0.282 \\
\hline Differentiated & $128(51.2 \%)$ & 57 (57.6\%) & \\
\hline Undifferentiated & $122(48.8 \%)$ & $42(42.4 \%)$ & \\
\hline
\end{tabular}

Table 1 (continued)
Table 1 (continued)

\begin{tabular}{|c|c|c|c|}
\hline \multirow{2}{*}{ Variable } & Training cohort & Validation cohort & \multirow{2}{*}{$\mathrm{P}$} \\
\hline & $n=250$ & $\mathrm{n}=99$ & \\
\hline With SRC & & & 0.402 \\
\hline No & $173(69.2 \%)$ & $73(73.7 \%)$ & \\
\hline Yes & 77 (30.8\%) & $26(26.3 \%)$ & \\
\hline TP (g/L) & $63.4 \pm 7.2$ & $62.8 \pm 7.7$ & 0.475 \\
\hline LSR & $0.85 \pm 0.32$ & $0.87 \pm 0.38$ & 0.626 \\
\hline ALKP (U/L) & 67 [48-72] & 63 [48-72] & $0.021^{*}$ \\
\hline LDH (U/L) & $183.3 \pm 45.4$ & $175.6 \pm 55.3$ & 0.182 \\
\hline Tbil $(\mu \mathrm{mol} / \mathrm{L})$ & $9.4(6.7-12.6)$ & $9.5(6.5-13.6)$ & 0.712 \\
\hline Dbil ( $\mu \mathrm{mol} / \mathrm{L})$ & $2.7(1.9-3.8)$ & $2.7(2.0-4.0)$ & 0.333 \\
\hline PT (s) & $11.4 \pm 0.9$ & $11.5 \pm 1.0$ & 0.626 \\
\hline APTT (s) & $29.6 \pm 3.2$ & $29.7 \pm 3.13$ & 0.748 \\
\hline$\pi(s)$ & $19.1 \pm 1.6$ & $19.3 \pm 1.8$ & 0.198 \\
\hline CEA (ng/mL) & $2.60(1.57-4.35)$ & $2.17(1.46-3.54)$ & 0.069 \\
\hline CA199 (U/mL) & $7.04(2.94-21.87)$ & $6.63(3.28-17.12)$ & 0.558 \\
\hline HPR & $0.51(0.34-0.64)$ & $0.51(0.34-0.69)$ & 0.794 \\
\hline HALP & $30.4(18.4-47.6)$ & 30.5 (17.7-44.8) & 0.544 \\
\hline
\end{tabular}

*, $\mathrm{P}<0.05$. SRC, signet ring cell; TP, total protein; LSR, alanine aminotransferase and aspartate aminotransferase ratio; ALKP, alkaline phosphatase; LDH, lactate dehydrogenase; PT, prothrombin time; APTT, activated partial thromboplastin time; TT, thrombin time; HPR, hemoglobin and platelet ratio; HALP, the hemoglobin, albumin, lymphocyte, and platelet. SRC, signet ring cell; TP, total protein; LSR, alanine aminotransferase and aspartate aminotransferase ratio; ALKP, alkaline phosphatase; LDH, lactate dehydrogenase; Tbil, total bilirubin; Dbil, direct bilirubin; PT, prothrombin time; APTT, activated partial thromboplastin time; TT, thrombin time; CEA, carcinoembryonic antigen; CA199, carbohydrate antigen 19-9; HPR, hemoglobin and platelet ratio; HALP, the hemoglobin, albumin, lymphocyte, and platelet.

Cut-off values obtained in ROC curve analysis according to Youden's J index, which were $11.1 \mathrm{~s}, 3.49 \mathrm{ng} / \mathrm{mL}$, $14.11 \mathrm{U} / \mathrm{mL}, 0.66$ and 35.3 (Figure 1, Table 2). After analysis, the results of the multivariate regression analysis showed that alcohol use ( $\mathrm{OR}=2.203$; 95\% CI: $1.054-4.605, \mathrm{P}=0.036)$, depth of invasion $(\mathrm{OR}=7.756 ; 95 \% \mathrm{CI}: 3.853-15.614$ $\mathrm{P}<0.001)$, differentiation ( $\mathrm{OR}=2.252$; 95\% CI: 1.149-4.416, $\mathrm{P}=0.018), \mathrm{CEA} \geq 3.49 \mathrm{ng} / \mathrm{mL}(\mathrm{OR}=2.443 ; 95 \% \mathrm{CI}: 1.175$ 5.080, $\mathrm{P}=0.017), \mathrm{CA} 199 \geq 14.11 \mathrm{U} / \mathrm{mL}(\mathrm{OR}=2.715 ; 95 \% \mathrm{CI}$ : 
1.292-5.703, $\mathrm{P}=0.008)$, and HALP $\leq 35.3(\mathrm{OR}=2.276$; $95 \%$ CI: $1.075-4.818, \mathrm{P}=0.032$ ) were independent risk factors for LNM of GC (Tables 3,4).

\section{Nomogram development and validation}

The risk of LNM of GC was predicted based on the six independent risk factors determine by multivariate

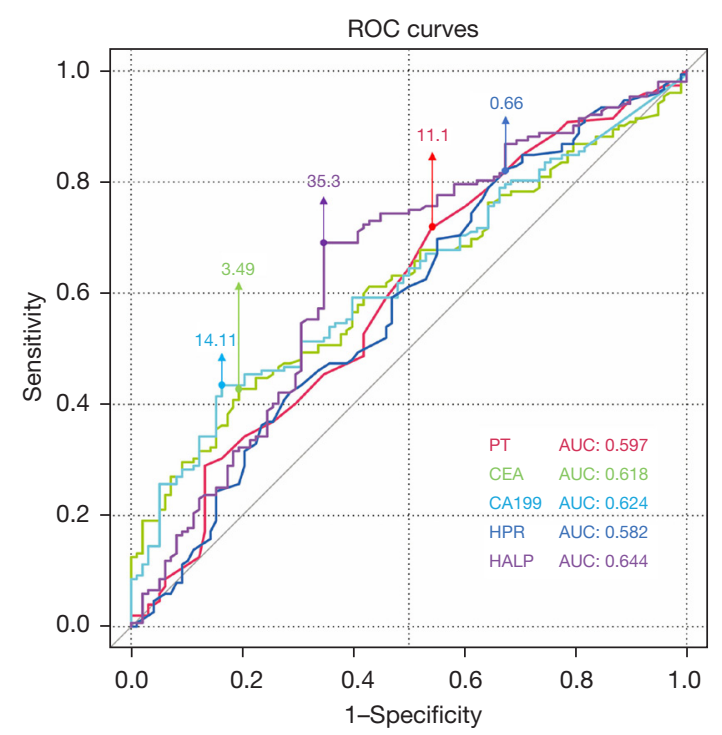

Figure 1 The ROC curve of PT, CEA, CA199, HPR and HALP for predicting patients with lymph node metastasis. PT, prothrombin time; HPR, hemoglobin and platelet ratio; HALP, the hemoglobin, albumin, lymphocyte, and platelet. ROC, receiver operating characteristic; PT, prothrombin time; CEA, carcinoembryonic antigen; CA199, carbohydrate antigen 199; HPR, hemoglobin and platelet ratio; HALP, the hemoglobin, albumin, lymphocyte, and platelet. analysis (Table 4). We chose these six factors to develop a predictive nomogram for LNM in GC patients (Figure 2). The first row ("Points") is used to assign a score for each variable below the first row by drawing a vertical line from the value for each variable to the "Points" line. The total points of patients were calculated by summing up all the separate points for the six predictors. The estimated incidence of LNM in GC patients can be finally determined by drawing a straight line down from the "Total Points" axis to the "Pr" axis. The scores of alcohol use, differentiation, CEA, CA199, and HALP were shown in Table 5, while Table 4 shows several examples of how to use the nomogram. The discriminative ability of the nomogram was assessed using the area under the curve (AUC), and the AUC for the training cohort and validation cohort were 0.854 (95\% CI: 0.802-0.906) and 0.868 (95\% CI: $0.798-0.968$ ) (Figure $3 A, 3 B$ ). The calibration plots presented a good agreement between the bias-corrected prediction and the ideal reference line with additional 1,000 bootstraps (Figure 4A,4B). DCA is a new method for assessing alternative prognostic strategies with significant advantages over AUC, and the DCA curves of nomogram in both the training and validation cohorts are shown in Figure $5 A, 5 B$. It can be seen that the line graph model shows a large positive net gain over a wide range of LNM risks in both cohorts, indicating that the model possesses good clinical validity.

\section{Discussion}

Currently, the main treatment strategies for GC are surgery and chemotherapy. According to the criteria for perigastric lymph node dissection published by the Japan Gastric Cancer Association (JGCA) in 2014 (15), the main surgical steps in radical GC surgery are radical resection of

Table 2 Diagnostic value of single indicators

\begin{tabular}{lcccccc}
\hline Variable & Sensitivity & Specificity & Cut-off & AUC & $95 \%$ Cl \\
\hline PT & $71.7 \%$ & $45.9 \%$ & 11.1 & 0.597 & $0.525-0.670$ & $0.009^{\star \star}$ \\
CEA & $42.8 \%$ & $80.6 \%$ & 3.49 & 0.618 & $0.549-0.686$ & $0.002^{\star \star}$ \\
CA199 & $43.4 \%$ & $83.7 \%$ & 14.11 & 0.624 & $0.555-0.692$ & $0.001^{\star \star}$ \\
HPR & $82.2 \%$ & $32.7 \%$ & 0.66 & 0.582 & $0.509-0.655$ & $0.029^{\star}$ \\
HALP & $69.1 \%$ & $65.3 \%$ & 35.3 & 0.644 & $0.572-0.715$ & $<0.001^{\star \star *}$ \\
\hline
\end{tabular}

${ }^{*}, \mathrm{P}<0.05 ;{ }^{* \star}, \mathrm{P}<0.01$; ${ }^{* \star}, \mathrm{P}<0.001$. PT, prothrombin time; HPR, hemoglobin and platelet ratio; HALP, the hemoglobin, albumin, lymphocyte, and platelet. AUC, area under the curve; PT, prothrombin time; CEA, carcinoembryonic antigen; CA199, carbohydrate antigen 19-9; HPR, hemoglobin and platelet ratio; HALP, the hemoglobin, albumin, lymphocyte, and platelet. 
Table 3 Univariate analysis of factors associated with lymph node metastasis

\begin{tabular}{|c|c|c|c|}
\hline \multirow{2}{*}{ Variable } & LNM(+) & LNM(-) & \multirow{2}{*}{$P$} \\
\hline & $n=152$ & $\mathrm{n}=98$ & \\
\hline Age (years) & $65.6 \pm 10.0$ & $63.5 \pm 10.2$ & 0.096 \\
\hline Gender & & & 0.275 \\
\hline Male & 115 (75.7\%) & $68(69.4 \%)$ & \\
\hline Female & $37(75.7 \%)$ & $30(30.6 \%)$ & \\
\hline Smoking & & & 0.753 \\
\hline No & $76(50.0 \%)$ & $51(52.0 \%)$ & \\
\hline Yes & $76(50.0 \%)$ & $47(48.0 \%)$ & \\
\hline Alcohol use & & & $0.007^{\star *}$ \\
\hline No & $95(62.5 \%)$ & 77 (78.6\%) & \\
\hline Yes & 57 (37.5\%) & $21(21.4 \%)$ & \\
\hline Diabetes & & & 0.294 \\
\hline No & $86(56.6 \%)$ & $62(63.3 \%)$ & \\
\hline Yes & $66(43.4 \%)$ & $36(36.7 \%)$ & \\
\hline $\begin{array}{l}\text { Coronary heart } \\
\text { disease }\end{array}$ & & & 0.604 \\
\hline No & 120 (78.9\%) & $80(81.6 \%)$ & \\
\hline Yes & $32(21.1 \%)$ & $18(18.4 \%)$ & \\
\hline Hypertension & & & 0.294 \\
\hline No & $86(56.6 \%)$ & $62(63.3 \%)$ & \\
\hline Yes & $66(43.4 \%)$ & $36(36.7 \%)$ & \\
\hline Location & & & 0.827 \\
\hline Upper $1 / 3$ & $21(13.8 \%)$ & $11(11.2 \%)$ & \\
\hline Middle 1/3 & $54(35.5 \%)$ & $35(35.7 \%)$ & \\
\hline Low $1 / 3$ & 77 (50.7\%) & $52(53.1 \%)$ & \\
\hline Weight loss & & & $0.004^{\star *}$ \\
\hline No & 79 (52.0\%) & $69(70.4 \%)$ & \\
\hline Yes & $73(48.0 \%)$ & $29(29.6 \%)$ & \\
\hline Depth of invasion & & & $<0.001^{\star \star *}$ \\
\hline $\mathrm{T} 1 / \mathrm{T} 2$ & $29(19.1 \%)$ & $71(72.4 \%)$ & \\
\hline $\mathrm{T} 3 / \mathrm{T} 4$ & 123 (80.9\%) & $27(27.6 \%)$ & \\
\hline Differentiations & & & $0.011^{*}$ \\
\hline Differentiated & $68(44.7 \%)$ & $60(61.2 \%)$ & \\
\hline Undifferentiated & $84(55.3 \%)$ & $38(38.8 \%)$ & \\
\hline
\end{tabular}

Table 3 (continued)
Table 3 (continued)

\begin{tabular}{|c|c|c|c|}
\hline \multirow{2}{*}{ Variable } & LNM(+) & LNM(-) & \multirow{2}{*}{$P$} \\
\hline & $n=152$ & $\mathrm{n}=98$ & \\
\hline \multicolumn{2}{|l|}{ With SRC } & & \multirow[t]{3}{*}{0.056} \\
\hline No & $99(65.1 \%)$ & 75 (76.5\%) & \\
\hline Yes & $53(34.9 \%)$ & $23(23.5 \%)$ & \\
\hline TP (g/L) & $63.1 \pm 7.4$ & $64.0 \pm 6.7$ & 0.302 \\
\hline LSR & $0.83 \pm 0.32$ & $0.89 \pm 0.33$ & 0.174 \\
\hline ALKP (U/L) & 67 [57-79] & 69 [58-79] & 0.271 \\
\hline LDH (U/L) & $179.0 \pm 45.7$ & $190.0 \pm 44.3$ & 0.062 \\
\hline Tbil (umol/L) & $9.6(6.8-12.8)$ & $9.2(6.5-12.5)$ & 0.866 \\
\hline Dbil (umol/L) & $2.9(2.0-3.9)$ & $2.5(1.8-3.4)$ & 0.050 \\
\hline PT (s) & $11.6 \pm 0.9$ & $11.3 \pm 0.9$ & $0.025^{\star}$ \\
\hline APTT (s) & $29.6 \pm 3.4$ & $29.6 \pm 3.0$ & 0.988 \\
\hline TT (s) & $18.9 \pm 1.7$ & $19.3 \pm 1.7$ & 0.076 \\
\hline CEA (ng/mL) & $2.95(1.84-5.47)$ & $2.24(1.47-3.27)$ & $0.002^{\star \star}$ \\
\hline CA199 (U/mL) & 9.24 (3.45-39.06) & $5.46(2.44-11.51)$ & $0.001^{\star \star}$ \\
\hline HPR & $0.49(0.33-0.61)$ & $0.56(0.40-0.71)$ & $0.028^{*}$ \\
\hline HALP & $27.2(15.8-41.0)$ & $38.8(20.7-54.7)$ & $<0.001^{\star * *}$ \\
\hline \multicolumn{4}{|c|}{$\begin{array}{l}{ }^{*}, \mathrm{P}<0.05 ;{ }^{* *}, \mathrm{P}<0.01 ;{ }^{* * *}, \mathrm{P}<0.001 . \mathrm{SRC} \text {, signet ring cell; TP, } \\
\text { total protein; LSR, alanine aminotransferase and aspartate } \\
\text { aminotransferase ratio; ALKP, alkaline phosphatase; LDH, } \\
\text { lactate dehydrogenase; PT, prothrombin time; APTT, activated } \\
\text { partial thromboplastin time; TT, thrombin time; HPR, hemoglobin } \\
\text { and platelet ratio; HALP, the hemoglobin, albumin, lymphocyte, } \\
\text { and platelet. LNM, lymph node metastasis; SRC, signet ring cell; } \\
\text { TP, total protein; LSR, alanine aminotransferase and aspartate } \\
\text { aminotransferase ratio; ALKP, alkaline phosphatase; LDH, } \\
\text { lactate dehydrogenase; Tbil, total bilirubin; Dbil, direct bilirubin; } \\
\text { PT, prothrombin time; APTT, activated partial thromboplastin } \\
\text { time; TT, thrombin time; CEA, carcinoembryonic antigen; CA199, } \\
\text { carbohydrate antigen 19-9; HPR, hemoglobin and platelet ratio; } \\
\text { HALP, the hemoglobin, albumin, lymphocyte, and platelet. }\end{array}$} \\
\hline
\end{tabular}

the lesion and perigastric lymph node dissection. Accurate assessment of preoperative lymph node status in patients with GC is critical. For patients with early GC without LNM, endoscopic mucosal resection and endoscopic submuscular dissection are widely accepted approaches that can not only preserve gastric function and maintain a high quality of life but also avoid post-operative complications caused by radical gastrectomy (16-18). For advanced GC, many oncologists recommend that NAC should be given to 
Table 4 Multivariate analysis of risk factors associated with lymph node metastasis

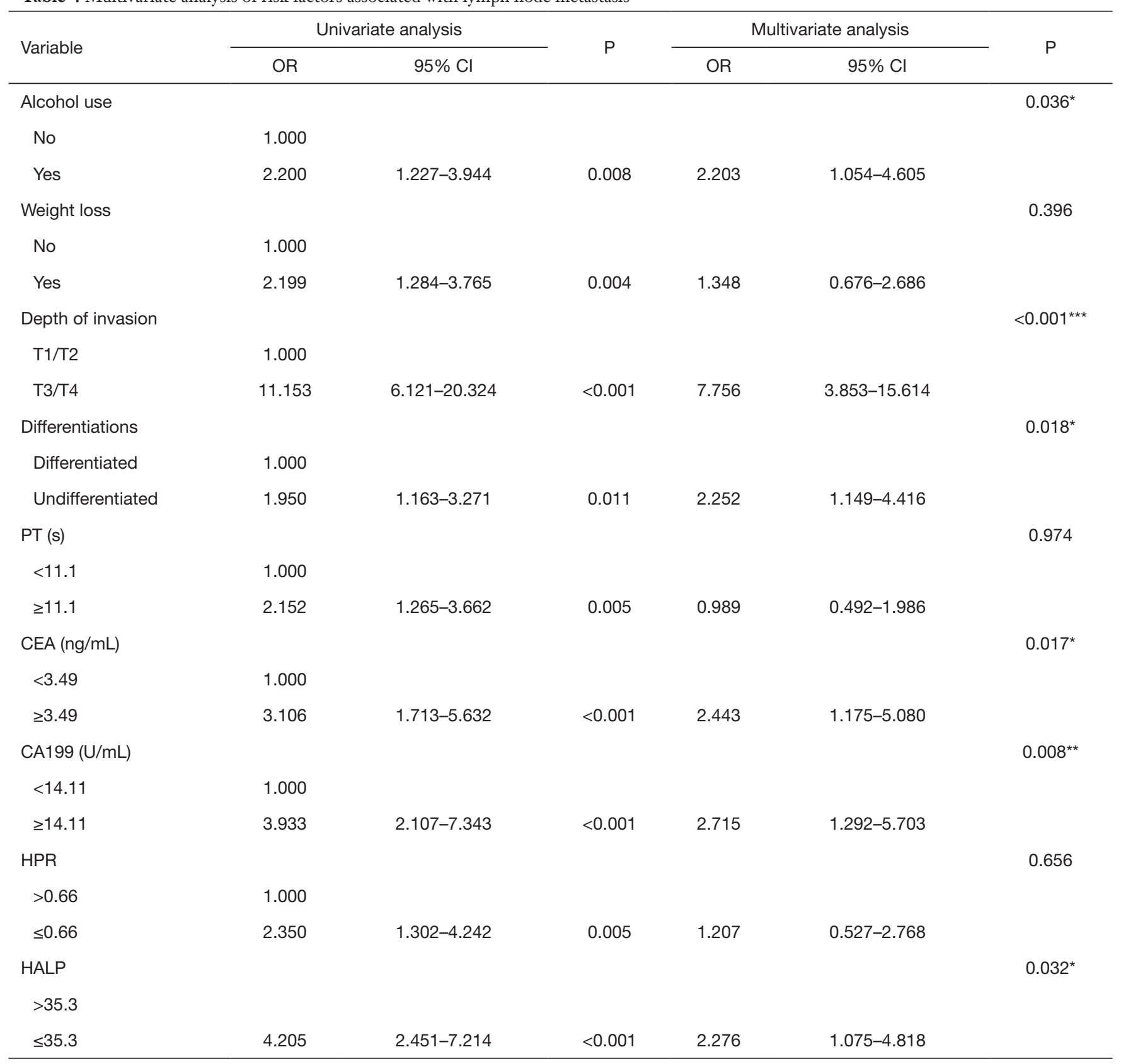

*, $\mathrm{P}<0.05 ;{ }^{* *}, \mathrm{P}<0.01 ;{ }^{* \star *}, \mathrm{P}<0.001$. PT, prothrombin time; HPR, hemoglobin and platelet ratio; HALP, the hemoglobin, albumin, lymphocyte, and platelet. PT, prothrombin time; CEA, carcinoembryonic antigen; CA199, carbohydrate antigen 19-9; HPR, hemoglobin and platelet ratio; HALP, the hemoglobin, albumin, lymphocyte, and platelet.

patients with advanced GC, especially those with LNM, to reduce preoperative TNM staging, thereby improving the rate of radical resection $(19,20)$. The results of this study may help to address this issue by combining independent risk factors, such as patient history, pathologic types, tumor markers and HALP score to predict the positive rate of LNM in patients with GC and thus develop a more individualized surgical approach.

The hemoglobin, albumin, lymphocyte and platelet (HALP) score are a comprehensive index that reflects components of the nutritional and immune status of patients, which has been shown to have a prognostic role 


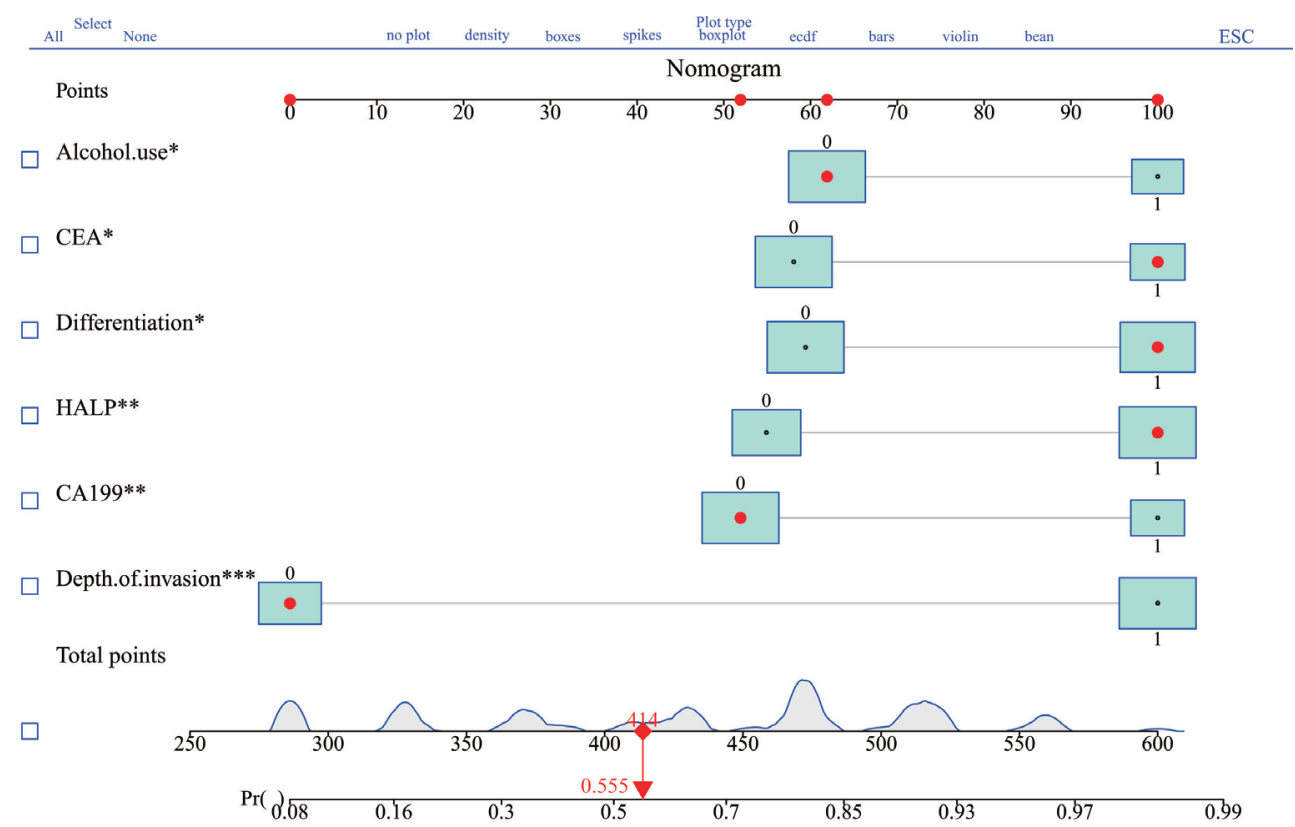

Figure 2 Nomogram for the prediction of lymph node metastasis in patients with gastric cancer. $\left(^{*}, \mathrm{P}<0.05 ;{ }^{* *}, \mathrm{P}<0.01 ;{ }^{* * *}, \mathrm{P}<0.001\right)$.

Table 5 Examples of lymph node metastasis risk prediction using the nomogram in patients with gastric cancer

\begin{tabular}{|c|c|c|c|c|c|c|c|c|}
\hline Variable & Group & Point & \multicolumn{2}{|c|}{ Patients-1 } & \multicolumn{2}{|c|}{ Patients-2 } & \multicolumn{2}{|c|}{ Patients-3 } \\
\hline \multirow[t]{2}{*}{ Alcohol use } & No $=0$ & 62 & & & No & 62 & No & 62 \\
\hline & Yes $=1$ & 100 & Yes & 100 & & & & \\
\hline Differentiation & $D=0$ & 59 & $\mathrm{D}$ & 59 & D & 59 & & \\
\hline \multirow[t]{2}{*}{ Depth of invasion } & $\mathrm{T} 1 / \mathrm{T} 2=0$ & 0 & $\mathrm{~T} 2$ & 0 & & & $\mathrm{~T} 1$ & 0 \\
\hline & $\mathrm{T} 3 / \mathrm{T} 4=1$ & 100 & & & $\mathrm{~T} 4$ & 100 & & \\
\hline \multirow[t]{2}{*}{ CEA } & $<3.49=0$ & 58 & 3.11 & 58 & 3.11 & 58 & & \\
\hline & $\geq 3.49=1$ & 100 & & & & & 9.55 & 100 \\
\hline \multirow[t]{2}{*}{ HALP } & $>35.3=0$ & 55 & 39.4 & 55 & & & & \\
\hline & $\leq 35.3=1$ & 100 & & & 24.5 & 100 & 14.5 & 100 \\
\hline Total points & & & & 372 & & 431 & & 414 \\
\hline Predicted & & & & 34.4 & & 64.2 & & 55.5 \\
\hline LNM risk (\%) & & & & & & & & \\
\hline
\end{tabular}

D, differentiated; UD, undifferentiated. D, differentiated; UD, undifferentiated; CEA, carcinoembryonic antigen; CA199, carbohydrate antigen 19-9; HALP, the hemoglobin, albumin, lymphocyte, and platelet; LNM, lymph node metastasis. 

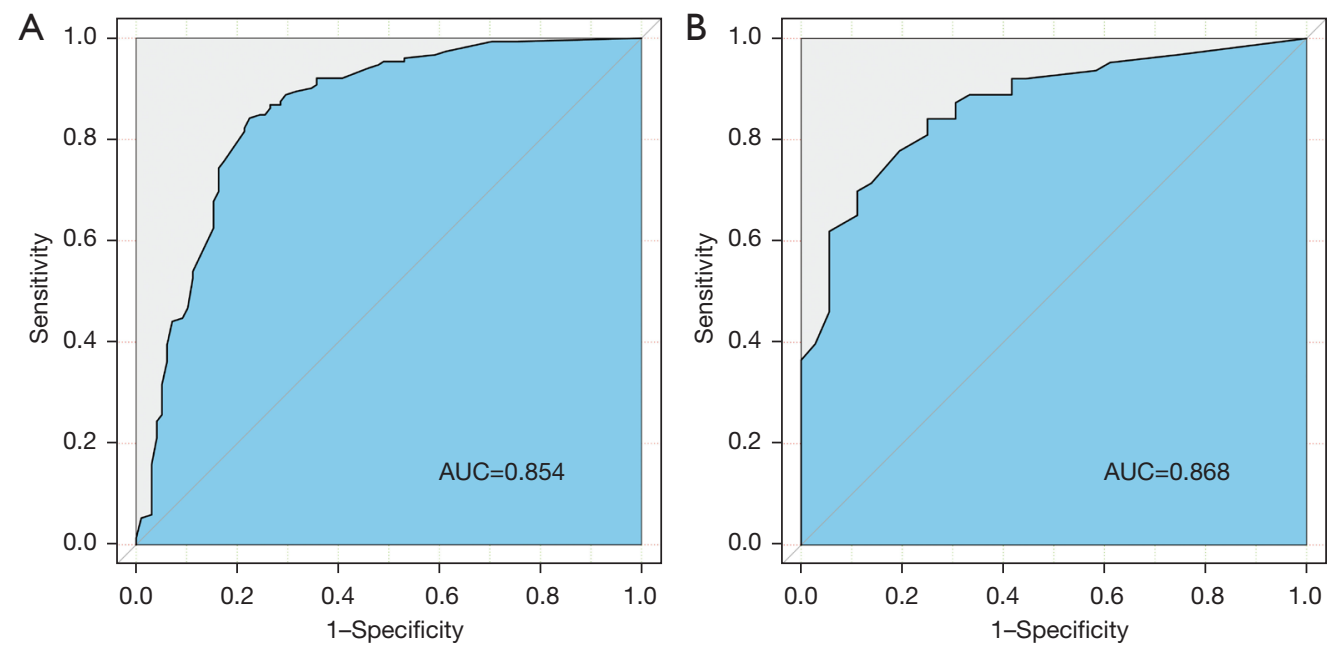

Figure 3 ROC plot of the nomogram (A) ROC plot of the nomogram in the training cohort. (B) ROC plot of the nomogram in the validation cohort. AUC, Area under the ROC curve.
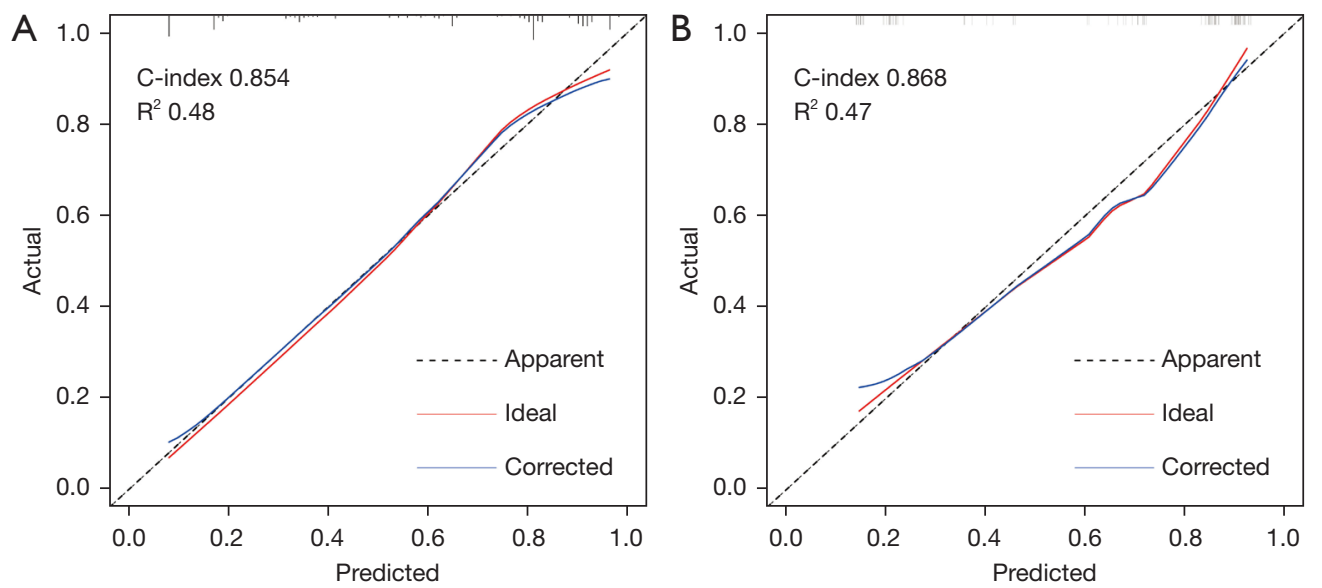

Figure 4 Calibration of the nomogram (A) Calibration plot in the training cohort. (B) Calibration plot in the validation cohort.

in gastrointestinal tumors and genitourinary tumors. Hemoglobin is a molecule which carries oxygen from respiratory organs to the rest of the body. Hypoxia is an important factor in tumor metabolism, survival, invasion, migration, angiogenesis and resistance to chemotherapy or radiation therapy (21). Due to their energetic needs and the hypoxia in the tumors, tumor cells need to adapt their metabolism, including glucose metabolism as they favor glycolysis, a phenomenon also known as the Warburg effect, which were frequently associated with the increased tumor cell aggressiveness and drug resistance (22). In addition, it has been recognized that the systemic inflammation response and nutrition status are significantly correlated with the progression and prognosis in GC $(23,24)$. Depletion of albumin due to cancer progression and decreased absorption of nutrients by the gastrointestinal tract due to GC are two possible causes of decreased albumin levels. In recent years, ALB has been considered not only as a nutritional marker, but also used to assess the inflammatory status of patients $(25,26)$, so that hypoalbuminemia may represent an increased inflammatory status in patients, which may lead to a poor prognosis $(27,28)$. Lymphocytes play an important role in recognizing tumor cells and antagonizing tumor, which may indirectly participate in inhibiting tumor cell proliferation as well as metastasis through immune surveillance and killing 

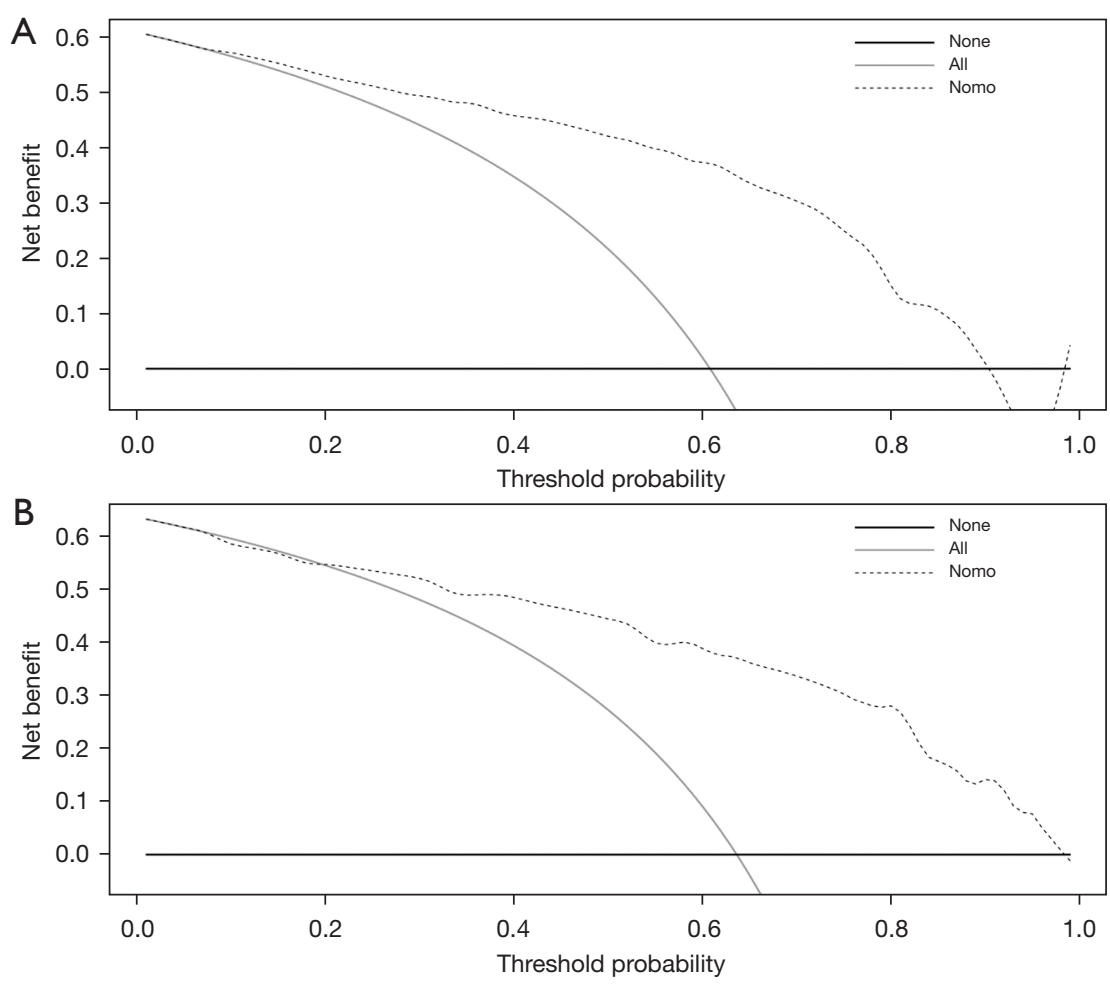

Figure 5 Decision curve analysis for the nomogram (A) Decision curve analysis of the nomogram for the prediction of lymph node metastasis in patients with gastric cancer in the training cohort. (B) Decision curve analysis of the nomogram for the prediction of lymph node metastasis in patients with gastric cancer in the validation cohort.

of tumor cells. The non-specific inflammatory response induced by malignant tumors is expected to elevate the platelet counts and lymphocytopenia in patients, and some studies have confirmed that the elevated platelet counts is associated with poor prognosis in patients with breast cancer and renal clear cell carcinoma (29-31). Platelets are associated with thrombosis and inflammation and release many cytokines, such as platelet-derived growth factor, platelet factor, platelet-reactive protein, vascular endothelial growth factor and thrombin sensitive protein-1, which have been shown to promote hematogenous tumor spread, tumor cell adhesion and invasion, and proliferation and to play an important role in angiogenesis of tumor tissue (32). Based on the above theory, high levels of hemoglobin, albumin and lymphocytes are favorable factors for survival and prognosis of tumor patients, while higher platelets were unfavorable factor. We calculated HALP scores based on hemoglobin level, albumin level, lymphocyte count and platelet count before the first treatment to reflect the systemic inflammation, immune and nutritional status of the organism. Our findings indicated that a low level of
HALP score (HALP $\leq 35.3$ ) was an independent risk factor for LNM in GC, which predicted an increased risk of LNM in GC.

Tumor markers (CEA and CA199) are classical and commonly used markers for GC (33). The present study also investigated the predictive value of CEA and CA199 for LNM in GC. It is noteworthy that the previous criteria for determining CEA and CA199 positivity (CEA $\geq 5.00 \mathrm{ng} / \mathrm{mL}$, CA199 $\geq 37.00 \mathrm{U} / \mathrm{mL}$ ) were not followed in this study, but the cut-off point was re-determined based on the ROC curve. The results of the study showed that high levels of CEA (CEA $\geq 3.14 \mathrm{ng} / \mathrm{mL})$ and CA199 (CA199 $\geq 14.11 \mathrm{U} / \mathrm{mL}$ ) were independent risk factors for LNM of GC. In this study, alcohol use was found to be an independent risk factor for LNM of GC, but no specific study revealed the mechanism involved. The possible underlying mechanism is that alcohol can damage the epithelium of gastric mucosa, resulting in the cancerous material eroding the epithelial tissues and gradually developing into malignant lesions. In addition, long-term alcohol consumption can lead to violation of the protein 
synthesis function of liver, followed by the development of lower nutritional levels. This study also found that the degree of tumor differentiation and depth of infiltration were independent risk factors for LNM, which showed great similarity with the results of other studies (34-36).

Nomograms have been widely used for quantifying the risk factors of LNM in various cancers $(37,38)$. Some previous studies have used information that is difficult to obtain preoperatively. In our study, history of alcohol consumption, CEA, CA199 and HALP score were easily obtained preoperatively. The degree of differentiation and depth of infiltration measured by gastroscopic biopsy and ultrasound gastroscopy had excellent accuracy. This nomogram model is easy to apply and has been proven to be highly effective in predicting LNM in patients with GC. Endoscopic mucosal resection or endoscopic submucosal dissection should be performed with caution in patients with early GC at very high risk of LNM, while NAC is more likely to be given to improve radical resection rates in patients with advanced GC at very high risk of LNM.

This research has certain limitations. On the one hand, this was a single-center retrospective study with a small sample size, which affected the accuracy of the results to some extent. On the other hand, the degree of tumor differentiation and depth of infiltration were all obtained postoperatively. Although they can also be obtained preoperatively by gastroscopic biopsy and ultrasound gastroscopy, a more in-depth study is needed to add some variabilities.

\section{Acknowledgments}

Funding: This work was supported by grants from Tianjin Science and Technology Bureau Major Disease Prevention and Control Technology Major Special Project (No. 19ZXDBSY00020), the National Natural Science Foundation of China (81900487) and the National Key R\&D Program of China (2019YFC0119505).

\section{Footnote}

Reporting Checklist: The authors have completed the TRIPOD reporting checklist. Available at https://dx.doi. org/10.21037/jgo-21-507

Data Sharing Statement: Available at https://dx.doi. org/10.21037/jgo-21-507
Conflicts of Interest: All authors have completed the ICMJE uniform disclosure form (available at https://dx.doi. org/10.21037/jgo-21-507). The authors have no conflicts of interest to declare.

Ethical Statement: The authors are accountable for all aspects of the work in ensuring that questions related to the accuracy or integrity of any part of the work are appropriately investigated and resolved. The study was conducted in accordance with the Declaration of Helsinki (as revised in 2013). The study was approved by the Ethical Committee of Tianjin Medical University General Hospital (No.: IRB2021-WZ-114) and individual consent for this retrospective analysis was waived.

Open Access Statement: This is an Open Access article distributed in accordance with the Creative Commons Attribution-NonCommercial-NoDerivs 4.0 International License (CC BY-NC-ND 4.0), which permits the noncommercial replication and distribution of the article with the strict proviso that no changes or edits are made and the original work is properly cited (including links to both the formal publication through the relevant DOI and the license). See: https://creativecommons.org/licenses/by-nc-nd/4.0/.

\section{References}

1. Bray F, Ferlay J, Soerjomataram I, et al. Global cancer statistics 2018: GLOBOCAN estimates of incidence and mortality worldwide for 36 cancers in 185 countries. CA Cancer J Clin 2018;68:394-424.

2. Jin C, Shi W, Wang F, et al. Long non-coding RNA HULC as a novel serum biomarker for diagnosis and prognosis prediction of gastric cancer. Oncotarget 2016;7:51763-72.

3. Rawicz-Pruszyński K, Ciseł B, Mlak R, et al. The Role of the Lymph Node Ratio in Advanced Gastric Cancer After Neoadjuvant Chemotherapy. Cancers (Basel) 2019;11:1914.

4. Dicken BJ, Bigam DL, Cass C, et al. Gastric adenocarcinoma: review and considerations for future directions. Ann Surg 2005;241:27-39.

5. Abdelfatah $M M$, Barakat $M$, Lee $H$, et al. The incidence of lymph node metastasis in early gastric cancer according to the expanded criteria in comparison with the absolute criteria of the Japanese Gastric Cancer Association: a systematic review of the literature and meta-analysis. Gastrointest Endosc 2018;87:338-47. 
6. Katai H, Ishikawa T, Akazawa K, et al. Five-year survival analysis of surgically resected gastric cancer cases in Japan: a retrospective analysis of more than 100,000 patients from the nationwide registry of the Japanese Gastric Cancer Association (2001-2007). Gastric Cancer 2018;21:144-54.

7. Kwee RM, Kwee TC. Imaging in assessing lymph node status in gastric cancer. Gastric Cancer 2009;12:6-22.

8. Huang KH, Lan YT, Fang WL, et al. The correlation between miRNA and lymph node metastasis in gastric cancer. Biomed Res Int 2015;2015:543163.

9. Li W, Ye F, Wang D, et al. Protein predictive signatures for lymph node metastasis of gastric cancer. Int J Cancer 2013;132:1851-9.

10. Zhang LX, Wei ZJ, Xu AM, et al. Can the neutrophillymphocyte ratio and platelet-lymphocyte ratio be beneficial in predicting lymph node metastasis and promising prognostic markers of gastric cancer patients? Tumor maker retrospective study. Int J Surg 2018;56:320-7.

11. Pang W, Lou N, Jin C, et al. Combination of preoperative platelet/lymphocyte and neutrophil/lymphocyte rates and tumor-related factors to predict lymph node metastasis in patients with gastric cancer. Eur J Gastroenterol Hepatol 2016;28:493-502.

12. Lou N, Zhang L, Chen XD, et al. A novel scoring system associating with preoperative platelet/lymphocyte and clinicopathologic features to predict lymph node metastasis in early gastric cancer. J Surg Res 2017;209:153-61.

13. Chen XL, Xue L, Wang W, et al. Prognostic significance of the combination of preoperative hemoglobin, albumin, lymphocyte and platelet in patients with gastric carcinoma: a retrospective cohort study. Oncotarget 2015;6:41370-82.

14. Japanese Gastric Cancer Association. Japanese classification of gastric carcinoma: 3rd English edition. Gastric Cancer 2011;14:101-12.

15. Japanese Gastric Cancer Association. Japanese gastric cancer treatment guidelines 2014 (ver. 4). Gastric Cancer 2017;20:1-19.

16. Saragoni L, Morgagni P, Gardini A, et al. Early gastric cancer: diagnosis, staging, and clinical impact. Evaluation of 530 patients. New elements for an updated definition and classification. Gastric Cancer 2013;16:549-54.

17. Baptista V, Singh A, Wassef W. Early gastric cancer: an update on endoscopic management. Curr Opin Gastroenterol 2012;28:629-35.

18. Lee HH, Yoo HM, Song KY, et al. Risk of limited lymph node dissection in patients with clinically early gastric cancer: indications of extended lymph node dissection for early gastric cancer. Ann Surg Oncol 2013;20:3534-40.

19. Cunningham D, Allum WH, Stenning SP, et al. Perioperative chemotherapy versus surgery alone for resectable gastroesophageal cancer. N Engl J Med 2006;355:11-20.

20. Ychou M, Boige V, Pignon JP, et al. Perioperative chemotherapy compared with surgery alone for resectable gastroesophageal adenocarcinoma: an FNCLCC and FFCD multicenter phase III trial. J Clin Oncol 2011;29:1715-21.

21. Peng X, Gao H, Xu R, et al. Correction to: The interplay between HIF-1 $\alpha$ and noncoding RNAs in cancer. J Exp Clin Cancer Res 2020;39:45.

22. Zhao Y, Butler EB, Tan M. Targeting cellular metabolism to improve cancer therapeutics. Cell Death Dis 2013;4:e532.

23. Balkwill F, Mantovani A. Inflammation and cancer: back to Virchow? Lancet 2001;357:539-45.

24. Sachlova M, Majek O, Tucek S. Prognostic value of scores based on malnutrition or systemic inflammatory response in patients with metastatic or recurrent gastric cancer. Nutr Cancer 2014;66:1362-70.

25. Mirsaeidi M, Omar HR, Sweiss N. Hypoalbuminemia is related to inflammation rather than malnutrition in sarcoidosis. Eur J Intern Med 2018;53:e14-6.

26. Hübner M, Mantziari S, Demartines N, et al. Postoperative Albumin Drop Is a Marker for Surgical Stress and a Predictor for Clinical Outcome: A Pilot Study. Gastroenterol Res Pract 2016;2016:8743187.

27. Kim S, McClave SA, Martindale RG, et al. Hypoalbuminemia and Clinical Outcomes: What is the Mechanism behind the Relationship? Am Surg 2017;83:1220-7.

28. Lohsiriwat V, Chinswangwatanakul V, Lohsiriwat $\mathrm{S}$, et al. Hypoalbuminemia is a predictor of delayed postoperative bowel function and poor surgical outcomes in right-sided colon cancer patients. Asia Pac J Clin Nutr 2007;16:213-7.

29. Zhang X, Zhao W, Yu Y, et al. Clinicopathological and prognostic significance of platelet-lymphocyte ratio (PLR) in gastric cancer: an updated meta-analysis. World J Surg Oncol 2020;18:191.

30. Zhu Y, Si W, Sun Q, et al. Platelet-lymphocyte ratio acts as an indicator of poor prognosis in patients with breast cancer. Oncotarget 2017;8:1023-30. 
31. Gunduz S, Mutlu H, Tural D, et al. Platelet to lymphocyte ratio as a new prognostic for patients with metastatic renal cell cancer. Asia Pac J Clin Oncol 2015;11:288-92.

32. Sierko E, Wojtukiewicz MZ. Platelets and angiogenesis in malignancy. Semin Thromb Hemost 2004;30:95-108.

33. Webb A, Scott-Mackie P, Cunningham D, et al. The prognostic value of serum and immunohistochemical tumour markers in advanced gastric cancer. Eur J Cancer 1996;32A:63-8.

34. Chen S, Nie RC, OuYang LY, et al. Nomogram analysis and external validation to predict the risk of lymph node metastasis in gastric cancer. Oncotarget 2017;8:11380-8.

35. Jiang Y, Wang W, Chen C, et al. Radiomics Signature on Computed Tomography Imaging: Association With Lymph Node Metastasis in Patients With Gastric Cancer.

Cite this article as: Wang $\mathrm{X}, \mathrm{He} \mathrm{Q}$, Liang $\mathrm{H}$, Liu J, $\mathrm{Xu} \mathrm{X}$, Jiang K, Zhang J. A novel robust nomogram based on preoperative hemoglobin and albumin levels and lymphocyte and platelet counts (HALP) for predicting lymph node metastasis of gastric cancer. J Gastrointest Oncol 2021;12(6):27062718. doi: 10.21037/jgo-21-507
Front Oncol 2019;9:340.

36. Huang C, Hu C, Zhu J, et al. Establishment of Decision Rules and Risk Assessment Model for Preoperative Prediction of Lymph Node Metastasis in Gastric Cancer. Front Oncol 2020;10:1638.

37. Dingemans SA, de Rooij PD, van der Vuurst de Vries RM, et al. Validation of Six Nomograms for Predicting Non-sentinel Lymph Node Metastases in a Dutch Breast Cancer Population. Ann Surg Oncol 2016;23:477-81.

38. Briganti A, Larcher A, Abdollah F, et al. Updated nomogram predicting lymph node invasion in patients with prostate cancer undergoing extended pelvic lymph node dissection: the essential importance of percentage of positive cores. Eur Urol 2012;61:480-7. 